SHORT REPORT

\title{
An audit to assess the quality of necropsies performed on stillborn infants
}

\author{
H Burnley, I Moore
}

J Clin Pathol 2004;58:93-94. doi: 10.1136/icp.2004.020636

Aims: To determine the quality of stillbirth postmortem reports and their contribution to a final diagnosis following the introduction of explicit consent forms after the Alder Hey inquiry.

Methods: Necropsy reports from 100 consecutive stillbirths were reviewed from 2001 onwards. A spreadsheet compiled data items that were considered essential in the Royal College of Pathologists guidelines. The type of consent (with permission for organs/tissue retention) was recorded to assess the impact on establishing a cause of death.

Results: Consent for tissue retention was obtained in 95 cases, whereas consent for organ retention was significantly lower (52 cases). In two cases, permission was refused for tissue retention and three requested external examinations only. Of these five, four had an undetermined cause of death, compared with 35 of 95 cases where permission for tissue retention was granted. All data items considered essential were recorded in every report. In 65 cases, the necropsy provided useful information, helped clinical care, and addressed parental concerns.

Conclusions: There was no major impact of the type of necropsy consent on establishing a cause of death, apart from the case of limited necropsies without histological examination of tissue samples.

$\mathrm{S}$ tillbirth is the most common category of death reported to the Confidential Enquiry into Stillbirths and Deaths in Infancy (CESDI), accounting for nearly one third of deaths in infants up to 1 year old. ${ }^{1}$ In the Wigglesworth classification," the largest proportion comprised "unexplained" antepartum fetal deaths (2472; 71.3\%), with intrapartum related events present in $7.3 \%$ (253) of cases. ${ }^{3}$ Other classification systems report substantially lower rates of unexplained stillbirths by including a category of small for gestational age or fetal growth restriction. ${ }^{3}$ After the Alder Hey inquiry in 2000, many trusts introduced explicit necropsy consent forms, giving parents a choice of tissue/organ retention and the extent of necropsy procedures. This study audits the necropsy outcomes following these changes.

\section{METHODS}

The Southampton Regional Paediatric Pathology Service, UK performs approximately 300 necropsies/year and 25\% are stillbirths. Seventy five percent are referral cases from outside Southampton. Necropsy reports from 100 consecutive stillbirths were reviewed from 2001 onwards. A spreadsheet compiled data items that were considered essential in the Royal College of Pathologists (RCPath) guidelines ${ }^{45}$ (body weight, crown-rump and crown-heel lengths, head circumference, foot length, organ weights, histology, and placental examination). The type of consent (with permission for

\begin{tabular}{|lc|}
\hline Table 1 Cause of death & \\
\hline Cause & $\mathrm{N}$ \\
\hline Unascertained & 4 \\
Undetermined cause, hypoxic mode & 32 \\
Placental cause & 26 \\
Placental insufficiency, IUGR & 2 \\
Umbilical cord pathology, hypoxic mode & 7 \\
Chronic villitis & 11 \\
Fetomaternal haemorrhage/placental abruption/APH & 9 \\
Chorioamnionitis, hypoxic mode & 6 \\
Fetal cause & 6 \\
Chromosomal or other congenital abnormalities & 1 \\
Maternal cause & 1 \\
Group B streptococcal infection & 1 \\
Bicornuate uterus, hypoxic mode & 100 \\
Possible lupus anticoagulant & \\
Total & \\
\hline APH, antepartum haemorrhage; IUGR, intrauterine growth restriction. & \\
\hline
\end{tabular}

organs/tissue retention) was recorded to assess the impact on establishing a cause of death. Other items noted included referral centre, antepartum/intrapartum death, sex, presence of intrauterine growth restriction (IUGR), gestational age, singleton/multiple pregnancy, ancillary investigations, history, cause of death, and turnaround time. IUGR was recorded if the weight was below the 10th percentile for gestational age and the brain to liver weight ratio was $>4$ : 1. Cases were classified by cause of death, results of ancillary investigations were tabulated, and the usefulness of necropsy findings was evaluated.

\section{RESULTS}

There were 97 singleton and three twin gestations, with one third of deaths occurring after 37 weeks of gestation, including nine intrapartum deaths. The male to female ratio was one. All data items considered essential by the RCPath were recorded in every report, and in 52 cases the reports were issued within the three week guideline. One quarter of the parents consented to a full necropsy, including the retention of organs for diagnosis, research, and education; $27 \%$ permitted organ retention for diagnosis only; and $95 \%$ consented to tissue retention. Four requested a full necropsy but specific consent for organ retention was absent (a fault in the design of a particular consent form). Two requested a necropsy with no permission for tissue retention and three requested an external examination only. Of these five cases, four had an undetermined cause of death, compared with 35 of 95 cases where permission for tissue retention was given. Despite consent for organ retention in 52 cases, the pathologists considered it unnecessary to retain whole organs. The cause of death remained undetermined in 19 of

Abbreviations: CESDI, Confidential Enquiry into Stillbirths and Deaths in Infancy; IUGR, intrauterine growth restriction; RCPath, Royal College of Pathologists 
Table 2 Investigations performed (number of cases)

\begin{tabular}{llll}
\hline Type of investigation & Requested & Result & N \\
\hline Chromosomal analysis & 52 & Failed & 23 \\
& & Normal & 22 \\
& & Absent & 5 \\
Microbiology & Abnormal & 2 (trisomy 21 and 18) \\
& \multirow{2}{*}{56} & No growth & 20 \\
Virology & & PM flora & 26 \\
& \multirow{2}{*}{55} & Group B streptococcus & 10 (9 vaginal commensals) \\
& & No growth & 35 \\
& & Absent & 20 \\
PM, postmortem. & Abnormal results & 0 \\
\hline
\end{tabular}

52 cases with permission for organ retention compared with 20 of 43 cases with permission for tissue only retention. In the six cases where the placenta was not submitted, the cause of death was undetermined, whereas placental pathology provided an explanation of death in 55 of 94 cases.

There were 17 poor clinical histories-for example, "stillbirth at 28 weeks" was considered woefully inadequate. Forty three were uneventful pregnancies, five mothers had diabetes or developed gestational diabetes, 13 had hypertension, and one had a bicornuate uterus.

Forty eight stillbirths had features of IUGR, 44 were within normal limits and eight were large for dates ( $>$ 90th centile). Table 1 provides the placental, fetal, and maternal causes of death. Table 2 provides the contribution of ancillary investigations.

\section{DISCUSSION}

In 1999, 57.6\% stillbirths occurring in England, Wales, and Northern Ireland had a necropsy, whereas our region had the highest rate, at $75.3 \%,{ }^{3}$ and was the only region to meet the perinatal necropsy rate of $75 \%$ recommended by a joint working party of the Royal College of Pathologists and the Royal College of Obstetricians and Gynaecologists. ${ }^{6}$ The RCPath guidelines recommend that a report, including histology, should be ready within three weeks, ${ }^{4}$ but we failed to meet this target in 48 cases, partly as a result of the high workload. We recognise that this is a very stringent target and that most centres aim for six weeks to correspond with the clinical follow up visit. Our aim is to reduce turnaround times to meet the local users' expectations and another full time paediatric pathologist has recently been appointed. Thorton and O'Hara found that when the findings of additional investigations (cytogenetics, microbiology, and virology) formed part of the necropsy report, results were often absent. ${ }^{7}$ Thirteen of our cases had absent results (in some cases karyotyping results were reported directly to the clinical team). Virological studies provided no additional information, and in light of this audit, samples for virology will not be routinely taken from stillborn infants. The two positive cytogenetic results were on infants with numerous phenotypic abnormalities.

"In 65 cases information obtained from necropsy alone helped in clinical care and addressed parental concerns, despite limitations on the necropsy procedure imposed by the new consent forms"

Using a similar approach to Vujanic et al, 55 necropsies yielded a diagnosis, one confirmed a suspected clinical diagnosis, nine found additional non-diagnostic information, and in 35 no new information was provided. In summary, in 65 cases information obtained from necropsy alone helped in
Take home messages

- We investigated the effect of the introduction of explicit consent forms for necropsy after the Alder Hey inquiry

- For necropsies performed on stillborn infants we found that the type of necropsy consent had no major impact on establishing a cause of death, apart from limited necropsies without histological examination of tissue samples

clinical care and addressed parental concerns, despite limitations on the necropsy procedure imposed by the new consent forms. This figure is similar to the one published in the fifth CESDI report (representing a pre-Alder Hey approach), where no explanation or associated condition for the cause of death could be found in $31 \%$ of late third trimester antepartum stillbirths. ${ }^{9}$ We showed no major impact of the type of necropsy consent (apart from a limited necropsy with no histological examination) on the final categorisation of the cause of death.

\section{Authors' affiliations}

H Burnley, I Moore, Department of Paediatric Pathology, Mailpoint 002, Southampton University Hospitals NHS Trust, Tremona Road, Southampton SO16 6YD, UK

Correspondence to: Dr I Moore, Department of Paediatric Pathology, Mailpoint 002, Southampton University Hospitals NHS Trust, Tremona Road, Southampton SO16 6YD, UK; isabella.moore@suht.swest.nhs.uk

Accepted for publication 26 August 2004

\section{REFERENCES}

1 Executive summary of the 8th annual report. Confidential enquiry into stillbirths and deaths in infancy (http://www.cemach.org.uk).

2 Wigglesworth JS. Monitoring perinatal mortality-a pathophysiological approach. Lancet 1980;ii:684-6.

3 CESDI. 8th annual report: focusing on stillbirths, European comparisons of perinatal care, paediatric post-mortem issues, survival rates of premature babies-Project 27/28. London: Maternal and Child Health Research Consortium, 2001.

4 The Royal College of Pathologists. Guidelines for post-mortem reports. London, Royal College of Pathologists, 1993.

5 The Royal College of Pathologists. Guidelines on autopsy practice: further material for discussion. Scenario 12: stillborn infant (singleton). London: The Royal College of Pathologists, November, 2003.

6 Joint Working Party RCOG/RCPath. Report on fetal and perinatal pathology. London: Royal College of Obstetricians and Gynaecologists, 1988.

7 Thorton CM, O'Hara MD. A regional audit of perinatal and infant autopsies in Northern Ireland. Br J Obstet Gynaecol 1998;105:18-23.

8 Vujanic GM, Cartlidge PH, Stewart JH, et al. Perinatal and infant post-mortem examinations: how well are we doing? J Clin Pathol 1995;48:998-1001.

9 Confidential Enquiry into Stillbirths and Deaths in Infancy. Annual report, 1996. London: Department of Child Health, 1998. 